\title{
AVALIAÇÃO DA FUNCIONALIDADE DE FAMÍLIAS COM IDOSOS
}

Ana Tarcia Guimarães Paiva ${ }^{1}$, Maria Eliana Peixoto Bessa ${ }^{2}$, Geridice Lorna Andrade de Moraes $^{3}$, Maria Josefina da Silva $^{4}$, Rosy Denyse Pinheiro de Oliveira ${ }^{5}$, Arethusa Morais de Gouveia Soares ${ }^{6}$

RESUMO: O estudo teve como objetivo avaliar a funcionalidade da família com idoso utilizando o instrumento APGAR de família. Estudo transversal realizado com 80 idosos residentes, ou não, com seus familiares, em área de abrangência de unidades de saúde de Fortaleza, Estado do Ceará. Os resultados indicaram que, em elevado percentual, os idosos mostraram-se satisfeitos com seus familiares, porém, aqueles que residem sozinhos apresentaram menor percentual de satisfação. O percentual de famílias funcionalmente satisfatórias foi de 83,7\%. Por meio da utilização do instrumento APGAR, verificou-se que muitas famílias com idosos apresentaram boa funcionalidade familiar. Dessa forma, o APGAR de família pode contribuir como um instrumento de triagem para detectar famílias disfuncionais.

PALAVRAS-CHAVE: Relações familiares; Idoso; Satisfação pessoal.

\section{EVALUATION OF FUNCTIONALITY IN FAMILIES WITH ELDERLY MEMBERS}

ABSTRACT: The study aimed to evaluate the functionality of families with elderly members, using the APGAR family instrument. A transversal study was made of 80 elderly persons either resident or not with their families, in an area covered by health units in Fortaleza, in the state of Ceará. The results indicated that a high percentage of the elderly claim to be satisfied with their families, but that those living alone show a lower level of satisfaction. The percentage of families held to be functionally satisfactory was $83.7 \%$. Through use of the APGAR tool it was corroborated that many families with elderly members had good family functionality. In this way, a family APGAR score can contribute as a triage tool for detecting dysfunctional families.

KEYWORDS: Family relationships; Elderly; Personal satisfaction.

\section{EVALUACIÓN DE LA FUNCIONALIDAD DE FAMILIAS CON ANCIANOS}

RESUMEN: El estudio tuvo como objetivo evaluar la funcionalidad de la familia con anciano utilizando la herramienta APGAR de familia. Estudio transversal realizado con 80 ancianos residentes, o no, con sus familiares, en área abarcada por las unidades de salud de Fortaleza, estado de Ceará. Los resultados apuntaron que, en gran porcentual, los ancianos están satisfechos con sus familiares, pero aquellos que viven solos presentaron menor porcentual de satisfacción. El porcentual de familias funcionalmente satisfactorias fue de 83,7\%. A través de la utilización de la herramienta APGAR, se verificó que muchas familias con ancianos presentaron buena funcionalidad familiar. De esa forma, el APGAR de familia puede contribuir como un instrumento de selección para detectar familias disfuncionales.

PALABRAS CLAVE: Relaciones familiares; Anciano; Satisfacción personal.

\footnotetext{
${ }^{1}$ Enfermeira Assistencial do Hospital Geral de Fortaleza/CE.

${ }^{2}$ Enfermeira. Doutoranda no Programa de Pós-Graduação em Enfermagem da Universidade Federal do Ceará-UFC. Bolsista PROPAG-REUNI.

${ }^{3}$ Enfermeira da Estratégia Saúde da Família do Município de Fortaleza/CE. Doutoranda no Programa de Pós-Graduação em Enfermagem da UFC.

${ }^{4}$ Enfermeira. Doutora em Enfermagem. Professora do Programa de Pós-Graduação em Enfermagem da UFC.

${ }^{5}$ Enfermeira da Estratégia de Saúde da Família do Município de Capistrano/CE. Especialista em Enfermagem Obstétrica.

${ }^{6}$ Enfermeira da Estratégia Saúde da Família do Município de Fortaleza/CE.
}

\author{
Autor correspondente: \\ Maria Eliana Peixoto Bessa \\ Universidade Federal do Ceará \\ R. Graciliano Ramos, 331 60415-050 - Fortaleza-CE-Brasil \\ E-mail: elianapbessa@gmail.com
}

Recebido: $26 / 09 / 10$

Aprovado: 19/02/11 


\section{INTRODUÇÃO}

O envelhecimento é a etapa final do ciclo de vida. Nessa fase, podem surgir vários problemas de saúde relacionados às doenças crônico-degenerativas, bem como perdas sociais, afetivas e financeiras. $\mathrm{O}$ direito à saúde é uma garantia a todo cidadão, sendo esse direito assegurado pelo Estado conforme a Constituição Brasileira de 1988. No caso do idoso, são necessárias políticas públicas resolutas e consistentes devido ao alto custo do cuidado a pessoa nessa fase da vida ${ }^{(1)}$.

O Estatuto do Idoso estabelece no artigo $3^{\circ}$ :

É obrigação da família, da comunidade, da sociedade e do Poder Público assegurar ao idoso, com absoluta prioridade, a efetivação do direito à vida, à saúde, à alimentação, à educação, à cultura, ao esporte, ao lazer, ao trabalho, à cidadania, à liberdade, à dignidade, ao respeito e à convivência familiar e comunitária ${ }^{(2: 8)}$.

Na sociedade ocidental, com as rápidas mudanças na dinâmica e estrutura familiar, o idoso vem perdendo o seu papel e função sociais ${ }^{(3)}$. Diante desse contexto de ruptura e readaptação de vínculos entre os idosos e seus familiares, surgem os seguintes questionamentos: como estão estruturadas as famílias com idosos? Qual a funcionalidade da família, segundo a opinião do idoso?

Entende-se por funcionalidade da família o modo como esta é capaz de cumprir e harmonizar as funções essenciais, de forma apropriada à identidade e às tendências das famílias e de seus membros, de forma realista em relação aos perigos e oportunidades que prevalecem no meio social ${ }^{(4)}$.

A família é um dos elementos centrais no cuidado do idoso. Desse modo, os profissionais que atuam no contexto da Estratégia Saúde da Família, e de forma singular o enfermeiro, devem estar atentos à funcionalidade familiar, de modo a planejar o cuidado direcionado às suas demandas ${ }^{(5)}$.

Com o intuito de avaliar a funcionalidade das famílias foi desenvolvido, em 1978, o instrumento APGAR de família por Smilkstein, sendo traduzido e validado para o contexto brasileiro por Duarte, em $2001^{(6)}$. Sabendo da importância da família para o idoso, estudos que visam avaliar sua funcionalidade por meio do instrumento APGAR de família tornamse relevantes, uma vez identificados problemas, os profissionais de saúde podem intervir de forma mais eficaz nesse contexto.

Assim, o objetivo deste estudo foi avaliar a funcionalidade da família com idoso utilizando o instrumento APGAR de família.

\section{METODOLOGIA}

Trata-se de um estudo transversal, de caráter exploratório e descritivo, desenvolvido junto a famílias com idosos, residentes na área de atuação de um Centro de Saúde da Família da Secretaria Executiva Regional III (SER III) no Município de Fortaleza Ceará.

A município de Fortaleza é organizado em seis Secretarias Executivas Regionais (SER), cada uma com área delimitada e abrangendo vários bairros. As SER são unidades administrativas nas quais se concentram as principais áreas de atuação do governo municipal, quais sejam: saúde, educação, serviço social, emprego e renda, cultura e habitação. Elas são definidas de acordo com a população, considerando vias de acesso, serviços públicos, e características ambientais e sanitárias.

Para a realização desta pesquisa, foi escolhido, de forma intencional, um Centro de Saúde da Família (CSF) por ser cenário de prática dos alunos do Curso de Enfermagem/UFC e do Programa de Reorientação da Formação Profissional em Saúde- PRÓ-SAÚDE/ Enfermagem/UFC. Esse CSF conta com duas equipes da Estratégia Saúde da Família (ESF), sendo, para este estudo, escolhida aleatoriamente uma equipe, a qual tem uma cobertura aproximada de 1400 famílias.

A amostra foi composta por idosos residentes em 8 quarteirões selecionados de forma aleatória. Em cada quarteirão foram entrevistados 10 idosos, residentes, ou não, com suas famílias. O processo de seleção da primeira residência com idoso se deu por indicação do agente de saúde da área, e as demais foram indicadas pelo método de "bola de neve", no qual a primeira residência indica a seguinte e assim se sucede até alcançar o número desejado ${ }^{(7)}$. Tal procedimento foi empregado devido à desatualização do cadastro da unidade de saúde, portanto, não havia precisão do número de famílias com idosos. A amostra totalizou 80 idosos.

Os critérios de inclusão dos idosos selecionados foram: residir na área de abrangência dos 8 quarteirões previamente selecionados, ser capaz de responder os questionamentos, e não apresentar déficit cognitivo.

A coleta de dados ocorreu no período de setembro a outubro de 2009 , por meio de visitas domiciliárias, e utilizando-se instrumento para a coleta de dados 
sociodemográficos e referentes ao APGAR da família.

O acrônimo APGAR é proveniente da língua inglesa e procura responder a estes aspectos $\left.{ }^{(8)}: 1\right)$ Adaptation (Adaptação): como os recursos são compartilhados ou qual o grau de satisfação do membro familiar com a atenção recebida; 2) Partnership (Companheirismo): como as decisões são compartilhadas ou qual a satisfação do membro da família com a reciprocidade da comunicação familiar na resolução de problemas; 3) Growth (Desenvolvimento): como a promoção do crescimento é compartilhada e qual o nível de satisfação do membro da família com a liberdade disponível no ambiente familiar; 4) Affection (Afetividade): como são as interações emocionais entre os membros da família e a relação de intimidade num contexto familiar; 5) Resolve (Capacidade resolutiva): como o tempo é compartilhado e qual a satisfação do membro familiar com esse compartilhamento ${ }^{(8)}$.

Os gradientes de respostas são: sempre (2), algumas vezes (1) e nunca (0), com uma pontuação final entre 0 e 10, indicando o intervalo de 0 a 4 como elevada disfunção familiar; 5 a 6 como moderada disfunção familiar; 7 a 10 como boa funcionalidade familiar ${ }^{(8)}$.

As entrevistas foram realizadas nos domicílios, em ambiente isolado e, preferencialmente, sem a presença de familiares. Como o instrumento trata do relacionamento do idoso com os parentes mais próximos, foi adotado esse procedimento para minimizar vieses nas respostas dos entrevistados. Todos os participantes foram convidados a assinar ou imprimir as digitais no Termo de Consentimento Livre e Esclarecido de acordo com a resolução do CNS n. 196 de 1996. O projeto de pesquisa foi aprovado no COMEPE/UFC sob protocolo n. 206/09.

Os dados foram organizados em forma de tabelas construídas no software SPSS versão 18.0 e analisados segundo a estatística descritiva, tendo como suporte teórico estudos sobre família e sua funcionalidade.

\section{RESULTADOS}

\section{Caracterização dos entrevistados}

\section{Os idosos}

Dos 80 idosos entrevistados, $76,3 \%$ eram do sexo feminino e $23,7 \%$ eram do sexo masculino. Com relação à faixa etária, 38,7\% tinham entre 60 a 69 anos; $37,5 \%$ entre 70 a 79 anos; $21,3 \%$ de 80 a
89 anos e 2,5\% acima de 90 anos. Sessenta e cinco idosos (81,3\%) moravam acompanhados e $18,7 \%$ relataram morar sozinhos.

\section{Pessoas que residem com os idosos}

O total de pessoas que residem com os idosos entrevistados é de 241 , sendo $59,3 \%$ do sexo feminino e $40,7 \%$ do sexo masculino. Dentre estes residentes $11,7 \%$ são cônjuges, $41,3 \%$ são filhos, $26,6 \%$ são netos e $20,4 \%$ são outros parentes e amigos dos idosos.

Com relação às faixas etárias, $11,6 \%$ tinham de 0 a 11 anos, $8,3 \%$ de 12 a 18 anos, $23,7 \%$ de 19 a 30 anos, $32,8 \%$ de 31 a 50 anos, $6,2 \%$ de 51 a 60 anos e 17,4\% tinham acima de 60 anos de idade.

Pode-se perceber que $68,7 \%$ das famílias eram constituídas por até 3 membros na família; $28,8 \%$ entre 4 a 6 membros e apenas 2,5\% apresentavam 7 a 9 membros familiares.

Na percepção dos idosos, $86,8 \%$ das pessoas relacionam-se bem com eles; enquanto $12,4 \%$ possuem uma relação regular, e $0,8 \%$ tem uma relação ruim. A tabela 1 mostra a qualidade da relação dos membros da família em comparação com a idade das pessoas que residem com os idosos. Dos que se relacionam satisfatoriamente, a maioria $(29,5 \%)$ está entre 31 e 50 anos. As relações consideradas ruins estão nas familias em que os membros familiares do idoso têm acima de 60 anos.

Quando observados os percentuais em todos os grupos de idade e relações, as mais positivas estão nos grupos entre $19-30$ anos $(20,7 \%)$ e 31 a 50 anos $(29,5 \%)$.

\section{Idosos que residem sozinhos}

A avaliação deste item permitiu verificar como os idosos que residem sozinhos utilizam as redes sociais de apoio. Setenta e um por cento recorrem a outras pessoas que não são familiares, e apenas $28,5 \%$ recorre aos seus parentes, sendo estes distribuídos da seguinte forma: 5,7\% são filhos, $17,1 \%$ são filhas e 5,7\% são netos. A maioria $(91,4 \%)$ das pessoas que apóia esses idosos é do sexo feminino e apenas $8,6 \%$ são do sexo masculino.

Os idosos costumam recorrer às pessoas das seguintes faixas etárias: 12 a $18(2,8 \%) ; 19$ a 30 $(5,7 \%) ; 31$ a $50(42,9 \%) ; 51$ a $60(14,3 \%)$ e acima de $60 \operatorname{anos}(34,3 \%)$. 
Tabela 1 - Faixa etária dos membros das famílias e qualidade da relação com o idoso. Fortaleza, 2009

\begin{tabular}{lcccccc}
\hline Qualidade da relação & \multicolumn{7}{c}{ Faixa etária } \\
\hline & $0-11$ & $12-18$ & $19-30$ & $31-50$ & $51-60$ & Acima de 60 \\
Boa & $11,2 \%$ & $6,2 \%$ & $20,8 \%$ & $29,5 \%$ & $5,4 \%$ & $13,7 \%$ \\
Regular & $0,4 \%$ & $2,1 \%$ & $2,9 \%$ & $3,3 \%$ & $0,8 \%$ & $2,9 \%$ \\
Ruim & $0,0 \%$ & $0,0 \%$ & $0,0 \%$ & $0,0 \%$ & $0,0 \%$ & $0,8 \%$ \\
\hline
\end{tabular}

\section{APGAR de família}

O primeiro componente do APGAR de família se refere à adaptação e avalia com qual frequência a seguinte afirmativa é verdadeira: "Estou satisfeito, pois posso recorrer à minha família em busca de ajuda quando alguma coisa está me incomodando ou preocupando". Ele representa a satisfação do idoso com relação à assistência recebida quanto a recursos familiares. Na pesquisa, verificou-se que $66,3 \%$ dos idosos afirmaram que sempre, $26,3 \%$ algumas vezes e 7,4\% nunca estão satisfeitos.

O segundo componente se refere a companheirismo e compreende a satisfação do membro familiar com a reciprocidade nas comunicações familiares e na solução de problemas: "Estou satisfeito com a maneira com a qual minha família e eu conversamos e compartilhamos os problemas". Os que se consideraram sempre satisfeitos foram $71,3 \%, 20 \%$ algumas vezes e $8,7 \%$ nunca estiveram satisfeitos.

O terceiro componente avalia o desenvolvimento e representa a satisfação do idoso com a liberdade disponibilizada pela família para mudanças de papeis e para alcance de maturidade ou desenvolvimento emocional. Esse item busca, ainda, responder a seguinte indagação afirmativa: "Estou satisfeito com a maneira como minha família aceita e apoia meus desejos de iniciar ou buscar novas atividades e procurar novos caminhos ou direções". A resposta "sempre satisfeitos" representou $76,3 \%$ dos entrevistados, $17,5 \%$ afirmaram estar algumas vezes e $6,2 \%$ nunca estão satisfeitos.

$\mathrm{O}$ quarto componente se refere à afetividade representando a afirmativa: "Estou satisfeito com a maneira como minha família demonstra afeição e reage às minhas emoções, tais como raiva, mágoa ou amor". Indica a satisfação do membro familiar com a intimidade e as interações emocionais em seu contexto familiar. Sessenta e seis por cento relataram sempre estar satisfeitos, $28,8 \%$ algumas vezes e $5,2 \%$ nunca estão satisfeito.

O quinto componente, capacidade resolutiva, representa a satisfação do membro familiar com o tempo compartilhado entre eles e se manifesta pela afirmação: "Estou satisfeito com a maneira com a qual minha família e eu compartilhamos o tempo juntos". Neste aspecto, 77,5\% consideraram-se sempre satisfeitos, $13,8 \%$ algumas vezes e $8,7 \%$ nunca estão satisfeitos.

Após a avaliação do APGAR de família obtivemos a seguinte classificação das famílias: $8,8 \%$ com elevada disfunção familiar; 7,5\% com moderada disfunção familiar e $83,7 \%$ com boa funcionalidade familiar.

\section{DISCUSSÃO}

\section{Idosos e famílias}

A família contribui de forma decisiva para uma velhice bem sucedida uma vez que é colocada como a maior responsável pelo atendimento das demandas do idoso. Desse modo, conhecer a família dos idosos é fornecer subsídios para assistência de qualidade e um envelhecimento ativo ${ }^{(9)}$.

Entre os idosos entrevistados houve a predominância do sexo feminino, $76,25 \%$, esse percentual está acima do encontrado na população brasileira de mulheres idosas em geral ${ }^{(10)}$. Pode-se inferir que a mulher está mais presente no domicílio, portanto, é mais provável que seja esta a participar neste contexto ambiental.

Além disso, as mulheres representam a maioria da população idosa. Essa predominância é comprovada internacionalmente, sendo maior nos países desenvolvidos. A esse fenômeno dá-se o nome de feminilização da velhice ${ }^{(11)}$.

Com relação à faixa etária, a Organização das Nações Unidas costuma classificar os idosos como pré-idosos (55 a 64 anos); idosos jovens (65 a 79 anos; ou 60 a 69 anos na Ásia e região do Pacífico); idoso de idade avançada (acima de 75 ou 80 anos) ${ }^{(12)}$.

Pode-se afirmar que a faixa etária predominante entre os idosos pesquisados foi a dos idosos jovens, pois $38,75 \%$ tinham entre 60 e 69 anos e $37,5 \%$ tinham entre 70 e 79 anos. Observa-se, ainda, que há 
um aumento gradual dos idosos para as idades mais avançadas, tendo em vista que a população acima de 80 anos é a que mais cresce no mundo ${ }^{(10)}$.

Em relação aos parentes dos idosos, verifica-se a presença majoritária do sexo feminino e cujo ofício de cuidador é exercido por mulheres. Quando a mulher idosa adoece é a filha, em primeiro lugar, que cuida, a seguir vêm os familiares femininos e, por último, o marido. Estudos demonstram que quando o homem idoso adoece, quem primeiro cuida é a esposa e depois a filha e, por último, os demais familiares ${ }^{(13)}$.

Dentre os aspectos pesquisados, um ponto positivo foi o nível de satisfação dos idosos com os seus familiares, já que em todos os componentes do APGAR de família os entrevistados responderam de forma positiva, o que contribui para sua qualidade de vida e seu bem-estar. Ressalta-se que os idosos contam com a rede social de apoio familiar; isso demonstra que a família, apesar de todas as transformações sofridas, ainda é um esteio para o idoso ${ }^{(3)}$.

Verificou-se que, na população pesquisada, grande parcela das famílias com idosos era composta de 1 a 3 membros, das quais muitas estavam nos estágios iniciais de desenvolvimento familiar, complexificando seu funcionamento, inclusive no cuidado ao idoso. Assim, a média de membros nas famílias foi de 4,5, incluindo o(s) idoso(s), o que é relevante quando se planeja o cuidado ao idoso atribuindo à família a responsabilidade maior dessa tarefa. Há tendência de redução do tamanho da família, que passou da média de 3,6 pessoas, em 1996, para 3,2, em $2006^{(10)}$.

O significativo número de pessoas com mais de 60 anos que mora com idosos leva a que o cuidador do idoso com dependência seja outro idoso. Este último também tem limitações físicas e emocionais, o que pode comprometer o cuidado na saúde daquele que primeiro necessita $^{(14)}$.

\section{Idosos e as redes sociais de apoio}

Em virtude das modificações decorrentes do processo de envelhecimento e das conjunturas familiares é essencial estabelecer uma rede de cuidados para os idosos, de modo especial para aqueles que residem sozinhos. As funções das redes sociais são entendidas como sendo as de promover interações interpessoais, que ocorrem dentro dessas estruturas, sendo compreendidas por quatro aspectos:

1) apoio emocional, que envolve expressões de amor e afeição; 2) apoio instrumental ou material que se refere aos auxílios concretos como provimento de necessidades materiais em geral, ajuda para trabalhos práticos (limpeza de casa, preparação de refeição, provimento de transporte) e ajuda financeira; 3) apoio de informação que compreende aconselhamentos, sugestões, orientações que podem ser usadas para lidar com problemas e resolvê-los; e 4) interação social positiva que diz respeito à disponibilidade de pessoas com quem se divertir e relaxar ${ }^{(15: 2983)}$.

Essas redes de apoio podem ser classificadas em formal e informal. A rede de apoio formal corresponde a grupos sociais formados pela unidade básica de saúde, pelos profissionais de saúde e pelos serviços do Estado. A rede de apoio informal é constituída pelos vizinhos, a própria família e os amigos ${ }^{(16)}$. Esta última foi o tipo de rede encontrada nesta pesquisa.

\section{Funcionalidade da família: APGAR de família}

Por meio dos dados coletados com o instrumento APGAR de família percebeu-se uma boa funcionalidade das famílias entrevistadas $(83,7 \%)$, significando que a maioria dos idosos está sempre satisfeita com o atendimento de suas demandas nos itens avaliados. Isso justifica o porquê os idosos consideram as relação com demais membro da família como boa, em todas as faixas etárias.

Semelhante perfil foi encontrado em estudo com idosos participantes de um grupo de alfabetização de um município do interior paulista, no qual $87,1 \%$ apresentaram boa funcionalidade familiar, $8,6 \%$ apresentaram moderada disfunção familiar e 4,3\% apresentaram elevada disfunção familiar ${ }^{(17)}$.

Em um universo maior de idosos, os dados tenderam a divergir. Estudo realizado com 117 idosos dependentes, residentes em domicílio em uma cidade do interior da Região do Nordeste brasileiro, identificou que $46,15 \%$ apresentavam moderada disfunção familiar, enquanto $27,35 \%$ apresentavam elevada disfunção familiar ${ }^{(18)}$.

Dentre os idosos que residiam sozinhos e participaram do presente estudo, o percentual de elevada disfunção familiar foi $20 \%$, bastante elevado se considerados os que residiam com familiares, que foi de $6,2 \%$. O fato de morar só, para o idoso, tem sido associado a um decréscimo na qualidade de vida, agravamento da morbidade e, até mesmo, indicador de risco de mortalidade ${ }^{(19)}$.

Em determinadas situações ou períodos, a capacidade da família para o cuidado pode estar 
comprometida ou fragilizada. E, nessas condições, o idoso pode constituir-se num entrave à autonomia dos familiares, seja pelas demandas do cotidiano ou pela impossibilidade de encontrar, entre os familiares, um ou mais membros que se disponibilizem e se responsabilizem pelo cuidado do idoso ${ }^{(20)}$. Percebemos, em nossa experiência profissional, que os idosos carregam alimentam a expectativa de receber atenção e cuidados dos filhos e netos no momento em que perderem ou tiverem sua autonomia diminuída.

Como a família é essencial para o desenvolvimento de seus membros e no processo saúde-doença, é importante que a Enfermagem estabeleça vínculos e estimule laços de compromisso e de responsabilidade entre os familiares que residem com idosos e sua rede social de apoio. O mito da família ser, naturalmente, responsabilizada pelo cuidado de seus idosos perde força na sociedade atual. Os arranjos familiares nem sempre são favoráveis à permanência do idoso, assim, o profissional que atua junto a esses grupos deve ser capaz de avaliar a melhor forma de intervir em benefício da pessoa idosa ${ }^{(9)}$.

\section{CONSIDERAÇÕES FINAIS}

O estudo avaliou a funcionalidade da família com o idoso, utilizando o instrumento APGAR de família. Esse instrumento de triagem pode ser utilizado para detectar famílias disfuncionais que necessitam ser priorizadas pelas equipes de saúde.

Por meio da utilização do instrumento APGAR verificou-se que muitas famílias com idosos apresentaram boa funcionalidade, sem dificuldades de relacionamento com os demais membros da família, tendo a mulher papel primordial no cuidado. Esses dados tornam-se essenciais para o planejamento assistencial à pessoa idosa.

A rede de apoio do idoso é formada por parentes e não-parentes. Os entrevistados apresentaram relações amistosas com os componentes da rede, o que proporciona um melhor cuidado a estes.

As ações do enfermeiro, especialmente o da atenção básica, direcionadas ao contexto familiar, no sentido de estabelecer esquemas assistenciais mais efetivos e dinâmicos, poderão contribuir significativamente para a qualidade da assistência ao idoso e reduzir custos emocionais da própria família.

Por ser um instrumento com poucos quesitos e com questões muito amplas, sugere-se que outras pesquisas sejam realizadas utilizando o APGAR de família e outros instrumentos que complementem e aprofundem a temática, possibilitando estudos comparativos.

O presente estudo teve como limitação o número reduzido de idosos e a delimitação não aleatória da área, dificultando a generalização dos resultados. Como possibilidades, o instrumento se mostrou eficaz para fornecer subsídios para uma intervenção multidimensional na família com idosos.

\section{REFERÊNCIAS}

1. Gomes MA, Pereira MLD. Família em situação de vulnerabilidade social: uma questão de políticas públicas. Ciênc Saúde Colet. 2005;10(2):357-63.

2. Brasil. Lei n. 10.741, de 1 de outubro de 2003. Dispõe sobre o estatuto do idoso e dá outras providências. Diário Oficial da União [Internet] 3 out 2003 [acesso em 13 mar 2009]. Disponível: http://www.planalto.gov. br/ccivil/LEIS/2003/L10.741.htm

3. Silva MJ, Bessa MEP, Oliveira AMC. Tamanho e estrutura familiar de idosos residentes em áreas periféricas de uma metrópole. Cienc Enferm. 2004;10(1):31-9.

4. Szymanski H. Práticas educativas familiares: a família como foco de atenção psicoeducacional. Estud Psicol. 2004;21(2):5-16.

5. Drulla AG, Alexandre AMC, Rubel FI, Mazza VA. A visita domiciliar como ferramenta ao cuidado familiar. Cogitare Enferm. 2009;14(4):667-74.

6. Duarte YAO. Família: rede de suporte ou fator estressor. A ótica de idosos e cuidadores familiares [tese]. São Paulo (SP): Universidade de São Paulo; 2001.

7. Malhotra NK. Pesquisa de marketing: uma orientação aplicada. 4ªed. São Paulo: Bookman; 2004.

8. Ministério da Saúde (BR). Secretaria de Atenção à Saúde. Envelhecimento e saúde da pessoa idosa. Brasília: Ministério da Saúde; 2007. (Série Normas e Manuais - Cadernos de Atenção Básica, no 19).

9. Martins JJ, Nascimento ERP, Erdmann AL, Candemil MC, Belaver GM. O cuidado no contexto domiciliar: o discurso de idosos/familiares e profissionais. Rev Enferm UERJ. 2009;17(4):556-62.

10. IBGE. Indicadores sociodemográficos e de saúde no 
Brasil. Rio de Janeiro: IBGE; 2007. (Série Estudos e Pesquisas Informação Demográfica e Socioeconômica, n. 21).

11. Salgado CDS. Mulher idosa: a feminização da velhice. Estud Interdiscipl Envelhec. [Internet] 2002;4(1) [acesso em 08 set 2010]. Disponível: http://seer.ufrgs.br/index.php/RevEnvelhecer/article/ viewFile/4716/2642

12. Ministério da Saúde (BR). BVS. Idosos no mundo. [Internet] [acesso em 18 ago 2010]. Disponível: http:// bvsms.saude.gov.br/bvs/exposicoes/idoso/idosob.html

13. Lima LD, Marques JC. Relações interpessoais em famílias com portador da doença de Alzheimer. Rev Psicol. 2007;38(2):157-65.

14. Miranda LC. Vamos falar um pouco mais sobre o cuidador? 2009. [acesso em 30 abr 2010]. Disponível: http://www.cuidardeidosos.com.br/vamos-falar-umpouco-mais-sobre-o-cuidador/.

15. Rosa TEC, Benício MHD’A, Alves PCGP, Lebrão ML. Aspectos estruturais e funcionais do apoio social de idosos do município de São Paulo, Brasil. Cad Saúde Pública. 2007;23(12):2982-92.

16. Souza EA, Maraschin MS, Andrade OG. Rede de apoio ao idoso no município de Cascavel. In: $2^{\circ}$ Seminário Nacional Estado e Políticas Sociais no Brasil; 2005 Out. p.6; Cascavel, Brasil. Cascavel: UNIOESTE; 2005.

17. Pavarini SC, Tonon FL, Silva JMC, Mendiondo MZ, Baham EJ, Filizola CLA. Quem irá empurrar minha cadeira de rodas? A escolha do cuidador familiar do idoso. Rev Eletr Enf. [Internet] 2006;8(3) [acesso em 13 mar 2009]. Disponível: http://www.fen.ufg.br/revista/ revista8_3/pdf/v8n3a03.pdf.

18. Torres V, Reis LA, Fernandes MH. Qualidade de vida e fatores associados em idosos dependentes em uma cidade do interior do Nordeste. J Bras Psiquiatr. 2009;58(1):39-44.

19. Caldas CP. Envelhecimento com dependência: responsabilidades e demandas da família. Cad Saúde Pública. 2003;19(3):733-81.

20. Perlini NMOG, Leite MT, Furini AC. Em busca de uma instituição para a pessoa idosa morar: motivos apontados por familiares. Rev Esc Enferm USP. 2007;41(2):229-36. 